Methodology for the analysis and comparison of protocols for glycaemic control in intensive care

Suggested running title: Methodology to analyse glycaemic control protocols

Rocío Fernández-Méndez*, Sancho Rodríguez-Villar, Pablo F. Méndez, Richard Windle, Gary George Adams

\title{
Authors' information
}

* Rocío Fernández-Méndez, RN MPH $\mathrm{PhD}^{1}$ (corresponding author)

Faculty of Medicine and Health Sciences, University of Nottingham

Medical School (West Block), NG7 2RD, Nottingham (UK)

Email: rociofmendez.inv@gmail.com / rf421@,cam.ac.uk; Tel: +44 (0)1223 746466

Sancho Rodríguez-Villar, LMS PhD EDIC

Dep. of Critical Care, King's College Hospital NHS Foundation Trust, London (UK)

Pablo F. Méndez, BSc PhD

Dep. of Ecology, Estación Biológica de Doñana, Seville (Spain)

Richard Windle, $\mathrm{PhD}$

Faculty of Medicine and Health Sciences, University of Nottingham

Medical School (West Block), NG7 2RD, Nottingham (UK)

Gary George Adams, BSc (Hons) RGN PGDipAdEd PhD FRSC FRSB FHEA

Faculty of Medicine and Health Sciences, University of Nottingham

Medical School (West Block), NG7 2RD, Nottingham (UK)

Keywords: blood glucose, critical care, clinical protocols, guidelines, evaluation methodology, analytical framework.

\footnotetext{
${ }^{1}$ Present address: Department of Clinical Neurosciences, University of Cambridge Biomedical Campus, Block A, level 3. CB2 0QQ, Cambridge (UK)
} 


\section{Summary}

Rationale, aims and objectives: The practice of glycaemic control of critically ill patients admitted to intensive care units (ICUs) is guided by clinical management protocols, designed locally by the ICUs. These protocols differ significantly in their aims and methods. The aim of this study was to develop a standardised methodology for the systematic and objective analysis and comparison of protocols for glycaemic control implemented in any ICU. Method: The protocols for glycaemic control implemented in seven ICUs of a UK-based ICU network were analysed using techniques of inductive content analysis, through an open coding process and the framework method. This involved the identification and classification of protocol instructions for glycaemic control, as well as of the processes and decisions pertaining to each of these instructions. These were used to develop a framework for the structured and systematic description and comparison of the protocols' contents, and to develop a technique for the protocols' graphic visualisation. Results: The following elements were identified or developed: (a) 35 quantifiable variables and 11 non-quantifiable subjects that could be present in an ICU protocol for glycaemic control, to be used as a framework for the description and comparison of contents; (b) a technique for condensing a protocol into a single, comprehensive flowchart; (c) using these flowcharts, a method for assessing the complexity and comprehensiveness of the protocols. Conclusions: The methodology developed in this study will allow for any future work analysing the contents of glycaemic control protocols to be carried out in a structured and standardised way. This may be done either as a standalone study, or as the essential first step in any investigation on the impact of new protocols. In turn, the methodology will facilitate the performance of regional, national and international comparisons, demonstrating the usefulness of this study at a global scale. 


\section{Introduction}

Hyperglycaemia, hypoglycaemia and increased glycaemic variability are three domains of glycaemic control that have been associated with higher risks of poor outcomes in critically ill patients, including impaired wound healing, neuro-myopathy, sepsis, multiple organ failure, cardiac dysfunction and mortality [1]. Currently, available evidence about ideal glycaemic target ranges, and the safest and most effective methods of glycaemic control to achieve these in intensive care units (ICUs), is discrepant and conflicting [2-6]. This is a crucial barrier to the development of common quality standards for glycaemic control of the critically ill. Thus, the recommendations of existing national and international guidelines on glycaemic control in the ICU differ among each other [7-18].

As a consequence of the discrepant evidence and the lack of common guidelines and standards about the management of dysglycaemias in intensive care, ICUs implement their own locally developed clinical management protocols for glycaemic control [19-25]. Clinical management protocols have been defined as documents that "provide advice to decide about what clinical diagnostic and treatment steps should be taken (...)", including “(..$)$ documents relating only to drug doses and prescription timing for the treatment of specific medical conditions" [26]. For ease of reading, these will be referred to as protocols.

Numerous studies have been carried out comparing the effectiveness of local protocols for glycaemic control [27-30], but these studies usually lack the fundamental previous step of describing and comparing in detail the characteristics and instructions of the protocols under comparison. The few reported studies describing and comparing different protocols for glycaemic control found important variances between them [20,23]. However, these studies either dealt with general descriptions of the protocols' instructions, or they focused mainly on 
the protocols' insulin algorithms. There is a lack of studies that report detailed descriptions and comparisons of each of the protocols' instructions for the management of different glycaemic ranges. This is possibly due to the lack of a standardised, replicable and globally transferable method, that allows for systematic descriptions and comparisons of the contents of ICU protocols for glycaemic control, and which would make these analyses comprehensive and comparable across hospitals and internationally.

The aim of this study was to develop a specific methodology for the systematic quantitative and qualitative description and comparison of the contents of protocols for glycaemic control. The aim was for this methodology to be comprehensive, as well as flexible, so that it could be used in any future studies requiring or aiming to describe and compare such protocols. This was part of a larger study, known as GlyCon, carried out within seven ICUs of the Mid Trent Critical Care Network (MTCCN).

\section{Methods}

\section{Sample of protocols}

The MTCCN is a critical care network of ICUs in the UK that aims to provide common standards of care and protocols to all critically ill patients admitted within the geographical area that it covers [31]. The MTCCN's Service Improvement Group encourages and facilitates the sharing of local policies, procedures and guidelines among the staff of its ICUs [32], and accepted GlyCon as a proposed study for the group. The seven ICUs of the MTCCN that admit patients with the highest levels of care needs (requiring either advanced respiratory support alone or support for a minimum of two organs), accepted to participate in GlyCon study. 
Soft copies of the protocols for glycaemic control, which had been in place in the seven participating ICUs during the study period (2012-2013), were acquired from each of the units through the study's local collaborators. In this study, the protocols for glycaemic control were the sources of information, whereas data were to be collected from the contents of these protocols.

\section{Data collection and analyses}

Data were collected (extracted from the protocols) through open coding (labelling) of the protocols' content as described by Elo \& Kyngäs [33]. This allowed categories of data to emerge directly from the textual information $[34,35]$, i.e. to be inferred inductively. Through this procedure, codes (labels) were first assigned to the selected initial units of analysis. These were each of the text sentences, or clauses within sentences, written in the protocols. The codes were grouped into broader categories, and these codes and inferred categories were used to label subsequent units of analysis. Using the framework method [36], summarised data were included (charted) into a matrix of codes (columns) and protocols (rows). A final set of categories of data or subjects, which may be present in a protocol, was abstracted through systematic comparisons of the charted data across protocols. These were used to develop a framework to guide the description and comparison of the contents of protocols for glycaemic control.

A technique for condensing protocol instructions within a single detailed flowchart, and a method for assessing the complexity and comprehensiveness of the protocols using such flowcharts, was proposed. Details about this technique are described in the results section, as this was a newly developed method and, therefore, an outcome of this study. For this method, a distinction was made between "processes", "decisions" and "scenarios". A process referred to any activity that should be carried out for the management of a patient's glycaemic status. 
A decision referred to any situation that would require thinking and judging what process should be carried out. For example, conditional instructions, such as "the insulin infusion may need to be modified after 12 hours if blood glucose levels are unstable", involved a decision step. A scenario referred to a possible loop comprising a sequence of processes and decisions between one glycaemic measurement and the next.

\section{Ethical Considerations}

This study did not involve human subjects. The study had sponsorship from the University of Nottingham, and had the ethical approval from a UK NHS Research Ethics Committee (REC Reference number:14/EM/0177) and the Research and Development Department of the Research and Development Department of the Hospital Trust of each participating ICU.

\section{Results}

The outcomes of this study included: (a) the array of 'categories' that emerged from the analysis and, based on these, a list of quantifiable 'variables' (Table 1) and a list of nonquantifiable 'subjects' (Table 2) that can be present in protocols for glycaemic control; (b) a technique for condensing a protocol into a single, comprehensive flowchart (Figure 1); (c) using the flowchart, a method for assessing the complexity and completeness of the protocols (Figure 2 and Table 3).

\section{Protocol categories, variables and subjects}

The two broadest categories of data identified in the protocols were:

1) The method used for the protocol development. This category included metadata about when, how and by whom the protocol was developed, as well as the evidence on which the protocol was based, and for which target patients it was meant to be used. These could be either quantifiable characteristics or non-quantifiable features of the protocols. 
2) The protocol instructions for glycaemic control. This category included any statement describing how glycaemic control should be carried out. Within this category, three key features were identified to classify each instruction:

a) The aim of the instruction: Based on this feature, five 'sub-categories' were further identified, namely diagnostic, monitoring, treatment, organisational and mixed instructions. Diagnostic instructions referred to those instructions related to the definition of the glycaemic status of the patient. Monitoring instructions referred to any indications on how or when blood glucose should be monitored. Treatment instructions included any written directions on the administration of insulin, other medication or glucose solutions with the aim of affecting glycaemic levels. Organisational instructions were indications related to the organisation of the ICU team, division of roles, or administrative activities, related to the management of glycaemic control in the ICU. Instructions that could be classified in more than one category were considered as mixed type of instruction.

b) The glycaemic status to which the instruction referred. Depending on this status, four further 'sub-categories' were distinguished, namely instructions relevant to glycaemic levels below, within or above the protocol's glycaemic target range, as well as instructions that were relevant at all glycaemic levels.

c) The quantifiable nature of the instruction. Based on this, instructions could be classified as either quantifiable data (instructions that could be counted or transformed into categorical variables) or non-quantifiable information.

These categories of protocol instructions were used to create the list of quantifiable variables (Table 1, 35 variables in total) and the list of non-quantifiable subjects (Table 2, 11 subjects in total). 


\section{Protocol flowcharts and scenarios}

The proposed method to design protocol flowcharts, and describe them, includes the steps enumerated below. An example of how this would be done is illustrated in Figure 1 and Figure 2 .

1) Identify each of the glycaemic ranges considered by the protocol.

2) Identify all the instructions dealing with each of these glycaemic ranges, for any given glycaemic measurement, and classify them as either a process or a decision (as defined in the Methods section).

3) Depict these processes and decisions schematically in closed-loop flowcharts, in a way that each loop (scenario) starts and finishes with a glycaemic measurement (Figure 1 and Figure 2). Processes should be represented with a rectangle, decisions with a diamond, and outputs or data (such as glycaemic levels) with a parallelogram, following the International Organization for Standardization (ISO) 5807 norm [37]. An example of the resulting flowchart for one of the protocols included in GlyCon study is included in Figure 1.

4) Identify and count all glycaemic ranges considered by the flowchart.

5) Identify and count all scenarios considered by the flowchart.

6) Identify and count all processes and decisions proposed within each scenario.

7) Populate Table 3 with metrics about the above counts (totals, medians and ranges), and with the types of processes and decisions considered by the flowcharts.

The metrics in Table 3 can be used to describe the number and types of scenarios, and processes and decisions interacting within each scenario. This may then be used to compare the complexity and comprehensiveness of different protocols for glycaemic control. Data from Table 1 and Table 2 complement Table 3 for such descriptions. In this respect, it is 8 
useful to note that Grol et al. [38] described an instruction or recommendation as complex when it is composed of numerous different interacting elements, and includes a complex decision tree, besides other factors that could affect these elements.

\section{Discussion}

Current management of stress hyperglycaemia among ICUs varies across ICUs globally, as well as among ICUs within the same country [21,22]. We have developed a convenient and comprehensive methodology to facilitate the structured and systematic investigation of recommendations and instructions within clinical management protocols for glycaemic control in the ICU.

At present there are a number of guideline appraisal tools that aim to help in assessing the quality of guidelines [39]. These tools are generic instruments that assess whether guidelines follow the principles of evidence based medicine, their aims and methods of development, and their clarity and applicability. Amongst the most widely used tools is the Appraisal of Guidelines for Research \& Evaluation (AGREE) II instrument, which was developed to assess the quality of clinical guidelines, and whether they should be recommended for use in practice [40]. This instrument rates the level to which guidelines deal with relevant aspects including their scope and purpose, stakeholder involvement, rigour of development, clarity of presentation, applicability and editorial independence. The iCAHE Guideline Quality Checklist [41] was developed as a shorter alternative to the AGREE II instrument. However, as it has been noted in previous studies [26], these tools are not relevant to studies exploring specific content recommendations or specific instructions within guidelines. They do not provide a structured methodology for the thorough description and comparison of the contents and complexity of the specific instructions of a protocol, which was the aim of our 
study. Specifically, we aimed to develop a methodology for identifying, describing and comparing each of the specific instructions included in the clinical management protocols for glycaemic control of any ICU.

The methodology used in this study has important strengths which can be summarised as follows. The combination of techniques of inductive content analysis and the framework method led to the systematic identification of a well-structured set of categories of instructions for glycaemic control, which had not been identified before. In the proposed framework for the analysis of protocols, a number of non-quantifiable subjects were included with the aims not only of enriching the quantifiable summaries, but also to help in understanding the rationale for the instructions and other types of data that cannot be quantified, as well as to make the findings more context specific. Separating these instructions according to the glycaemic status, rather than by narrower glycaemic ranges, as well as according to the recommendation type, provided a framework within which the instructions of different protocols for glycaemic control can be fitted and compared. This makes the method universally applicable to the analysis of different protocols. The proposed method to represent and summarise protocols graphically, through flow charts, provides a visual representation of the events sequence, which helps gain a shared understanding of the processes and decisions involved in the management of all and each of the glycaemic ranges considered by each protocol. The visual representation also helps to easily describe and compare the complexity and completeness of the protocols.

Therefore, the set of tools developed in GlyCon will facilitate the performance of future stuides exploring the practice of glycaemic control in ICU, and will increase the comparability and transferability of such studies. These studies are crucial to enable clinicians and researchers have a better understanding about how dysglycaemias in the 10 
critically ill are currently being managed within the ICU, whether at the institutional, regional or international levels. Moreover, exploring the recommendations that guide the methods for glycaemic control implemented in the ICU should be an essential first step of any study looking at the impact of such methods, including their effectiveness, efficiency and safety.

\section{Conclusions:}

Future work analysing and comparing the contents of local protocols for glycaemic control in ICU is essential to enable the clinical and scientific communities understand how dysglycaemic events are currently being managed in intensive care. Furthermore, these studies should be a fundamental initial step of any impact (outcome) evaluation of these protocols. The proposed methodology, developed as part of GlyCon study, will allow for such future work to be carried out in a standardised and comparable way. In turn, this will facilitate the performance of national and international comparisons, demonstrating the usefulness of this study at a global scale. 


\section{Acknowledgements}

We would like to thank all local collaborators of GlyCon study in each of the participating ICUs, for sharing their local protocols and policies. We would like to thank the Mid Trent Critical Care Network, for endorsing GlyCon as a study of their Quality Improvement Group. This work was supported by Nottingham University Hospitals (NUH) Charity and the NUH Department of Research and Innovation (grant awarded to Dr Gary Adams), and the University of Nottingham School of Health Sciences director of research small grants. The funding body did not participate in the design of the study and collection, analysis, and interpretation of data or in writing the manuscript.

\section{References}

1. Krinsley JS. Understanding glycemic control in the critically ill: three domains are better than one. Intensive Care Med. 2011;37(3):382-384. doi:10.1007/s00134-010-2110-3

2. Van den Berghe G, Wouters P, Weekers F, et al. Intensive insulin therapy in critically ill patients. N Engl J Med. 2001;345(19):1359-1367. doi:10.1056/NEJMoa011300

3. Chase G, Shaw GM, Le Compte A, et al. Implementation and evaluation of the SPRINT protocol for tight glycaemic control in critically ill patients: a clinical practice change. Crit Care. 2008;12(2):R49. doi:10.1186/cc6868

4. NICE-SUGAR Study Investigators, Finfer S, Chittock DR, et al. Intensive versus conventional glucose control in critically ill patients. N Engl J Med. 2009;360(13):12831297. doi:10.1056/NEJMoa0810625

5. Preiser JC, Devos P, Ruiz-Santana S, et al. A prospective randomised multi-centre controlled trial on tight glucose control by intensive insulin therapy in adult intensive 
care units: the Glucontrol study. Intensive Care Med. 2009;35(10):1738-1748. doi:10.1007/s00134-009-1585-2

6. Brunkhorst FM, Engel C, Bloos F, et al. Intensive insulin therapy and pentastarch resuscitation in severe sepsis. $N$ Engl $J$ Med. 2008;358(2):125-139. doi:10.1056/NEJMoa070716

7. Dellinger RP, Levy MM, Rhodes A, et al. Surviving Sepsis Campaign: international guidelines for management of severe sepsis and septic shock, 2012. Intensive Care Med. 2013;39(2):165-228. doi:10.1007/s00134-012-2769-8

8. American Diabetes Association. Executive summary: Standards of medical care in diabetes--2012. Diabetes Care. 2014;37(Supplement_1):S4-S10. doi:10.2337/dc14S005

9. Moghissi ES, Korytkowski MT, DiNardo M, et al. American Association of Clinical Endocrinologists and American Diabetes Association consensus statement on inpatient glycemic control. Diabetes Care. 2009;32(6):1119-1131. doi:10.2337/dc09-9029

10. Australian Diabetes Society. Guidelines for Routine Glucose Control in Hospital. Australian Diabetes Society; 2012.

11. Australian Diabetes Society. Perioperative Diabetes Management Guidelines. Australian Diabetes Society; 2012.

12. SEMICYUC. Quality Indicators in Critically Ill Patients. Update 2011. Spanish Society of Intensive Care Medicine and Coronary Units-Spanish Society of Parenteral and Enteral Nutrition; 2011. 
13. Ichai C, Preiser JC. International recommendations for glucose control in adult non diabetic critically ill patients. Crit Care. 2010;14(5):R166. doi:10.1186/cc9258

14. JBDS-IP. Glycaemic Management during the Inpatient Enteral Feeding of Stroke Patients with Diabetes Joint British Diabetes. Joint British Diabetes Societies (JBDS) for inpatient care Group; 2012.

15. JBDS-IP. Management of Adults with Diabetes Undergoing Surgery and Elective Procedures: Improving Standards. Joint British Diabetes Societies (JBDS) for inpatient care Group; 2011.

16. JBDS-IP. The Use of Variable Rate Intravenous Insulin Infusion (VRIII) in Medical Inpatients. Joint British Diabetes Societies (JBDS) for inpatient care Group; 2014.

17. Lü Q, Tong N. Interpretation of Chinese Society of Endocrinology consensus statement on hyperglycemia management target in adult inpatients in China (中华医学会内分泌 学分会《中国成人住院患者高血糖管理目标专家共识》解读). $J$ Diabetes. 2013;5(4):416-420. doi:10.1111/1753-0407.12058

18. Bruno G, De Micheli A, Frontoni S, Monge L. Highlights from "Italian Standards of Care for Diabetes Mellitus 2009-2010.” Nutr Metab Cardiovasc Dis. 2011;21(4):302-314. doi:10.1016/j.numecd.2010.08.009

19. Cook CB, Kongable GL, Potter DJ, Abad VJ, Leija DE, Anderson M. Inpatient glucose control: a glycemic survey of 126 U.S. hospitals. J Hosp Med. 2009;4(9):E7-E14. doi:10.1002/jhm.533 
20. Paddle JJ, Eve RL, Sharpe K a. Changing practice with changing research: results of two UK national surveys of intensive insulin therapy in intensive care patients. Anaesthesia. 2011;66(2):92-96. doi:10.1111/j.1365-2044.2010.06603.x

21. Wilson M, Weinreb J, Soo Hoo GW, Hoo GWS. Intensive insulin therapy in critical care: a review of 12 protocols. Diabetes Care. 2007;30(4):1005-1011. doi:10.2337/dc06-1964

22. Tomlinson VH, Langley J, Meal AG, Adams GG. "Medioglycaemia": A new concept in glycaemic control in intensive care (ICU) units? J Diabetes Mellit. 2012;2(4):361-368. doi:10.4236/jdm.2012.24056

23. Mackenzie I, Ingle S, Zaidi S, Buczaski S. Tight glycaemic control: a survey of intensive care practice in large English hospitals. Intensive Care Med. 2005;31(8):1136. doi:10.1007/s00134-005-2677-2

24. Orban J, Scarlatti A, Lefrant J, et al. Management of glycaemia: An audit in 66 ICUs [Gestion de la glycemie: un audit dans 66 reanimations]. Ann Fr Anesthèsie Rèanimation. 2013;32:84-88. doi:10.1016/j.annfar.2012.12.002

25. Niven DJ, Rubenfeld GD, Kramer AA, Stelfox HT. Effect of Published Scientific Evidence on Glycemic Control in Adult Intensive Care Units. JAMA Intern Med. 2015;175(5):801. doi:10.1001/jamainternmed.2015.0157

26. Guo Y, Adelstein B-A, Rubin GL. Availability and development of guidelines in a tertiary teaching hospital. J Eval Clin Pract. 2007;13(4):632-638. doi:10.1111/j.13652753.2007.00857.x 
27. Zimmerman CR, Mlynarek ME, Jordan J a, Rajda C a, Horst HM. An insulin infusion protocol in critically ill cardiothoracic surgery patients. Ann Pharmacother. 2004;38(78):1123-1129. doi:10.1345/aph.1E018

28. Chant C, Mustard M, Thorpe KE, Friedrich JO. Nurse- vs Nomogram-Directed Glucose Control in a Cardiovascular Intensive Care Unit. Am J Crit Care. 2012;21(4):270-278. doi:10.4037/ajec2012713

29. Scheuren L, Baetz B, Cawley MJ, Fitzpatrick R, Cachecho R. Pharmacist Designed and Nursing-driven Insulin Infusion Protocol to Achieve and Maintain Glycemic Control in Critical Care Patients. J Trauma Nurs. 2006;13(3):140-145. doi:10.1097/00043860200607000-00015

30. Kanji S, Jones E, Goddard R, Meggison HE, Neilipovitz D. Efficiency and safety of a standardized protocol for intravenous insulin therapy in ICU patients with neurovascular or head injury. Neurocrit Care. 2010;12(1):43-49. doi:10.1007/s12028-009-9275-z

31. UK Department of Health. Comprehensive Critical Care. A Review of Adult Critical Care Services. London, UK; 2000.

32. Mid Trent Critical Care Network. Mid Trent Critical Care Network. Home. http://www.midtrentccn.nhs.uk/. Published 2011.

33. Elo S, Kyngäs H. The qualitative content analysis process. J Adv Nurs. 2008;62(1):107115. doi:10.1111/j.1365-2648.2007.04569.x

34. Hsieh HF, Shannon SE. Three Approaches to Qualitative Content Analysis. Qual Health Res. 2005;15(9):1277-1288. doi:10.1177/1049732305276687 
35. Phil J. Inductive Analysis. In: Thorpe R, Holt R, eds. The SAGE Dictionary of Qualitative Management Research. London: SAGE Publications Ltd; 2008:298-299. doi:10.4135/9780857020109

36. Gale NK, Heath G, Cameron E, Rashid S, Redwood S. Using the framework method for the analysis of qualitativelndata in multi-disciplinary health research. BMC Med Res Methodol. 2013;13:117.

37. ISO. ISO 5807: Information Processing-document Symbols and Conventions for Data, Program and System Flowcharts, Program Network Charts and System Resource Charts. Geneva; 1985.

38. Grol R, Dalhuijsen J, Thomas S, Rutten G, Mokkink H. Attributes of clinical guidelines that influence use of guidelines in general practice: observational study. $\mathrm{Br} \mathrm{Med} J$. $1998 ; 317: 858-861$.

39. Siering U, Eikermann M, Hausner E, Hoffmann-eßer W, Neugebauer EA. Appraisal Tools for Clinical Practice Guidelines: A Systematic Review. PloS One. 2013;8(12). doi:10.1371/journal.pone.0082915

40. Brouwers MC, Kho ME, Browman GP, et al. AGREE II: advancing guideline development, reporting and evaluation in health care. Can Med Assoc J. 2010;182(18):E839-E842. doi:10.1503/cmaj.090449

41. Grimmer K, Dizon J, Milanese S, et al. Efficient clinical evaluation of guideline quality: development and testing of a new tool. BMC Med Res Methodol. 2014;14(1):63. doi:10.1186/1471-2288-14-63 
Figure 1 - Example of one of the resulting flowcharts of protocol instructions. BG: blood glucose; IU: international units of insulin; h: hour

Figure 2 - Example of the scenario analysis of protocol flowcharts. Left: identification of one possible scenario (loop) from one glycaemic measurement within $4.0-6.1 \mathrm{mmol} / \mathrm{L}$ and the next glycaemic measurement. Right: identification of the numbers and types of processes and decisions within one scenario. BG: blood glucose; IU: international units of insulin; h: hour 
Table 1 - Quantifiable variables that could be present in protocols for glycaemic control, organised by instruction aim \& glycaemic status to which the instruction is relevant - template table for future studies.

\begin{tabular}{|c|c|c|c|c|c|}
\hline \multirow{2}{*}{$\begin{array}{l}\text { Glycaemic } \\
\text { Status }\end{array}$} & \multirow{2}{*}{$\begin{array}{l}\text { Instr. } \\
\text { Aim* }\end{array}$} & \multirow{2}{*}{ Quantifiable Variables } & \multicolumn{3}{|c|}{ Protocol ID } \\
\hline & & & $\mathbf{1}^{[\mathrm{j}]}$ & 2 & Etc. \\
\hline \multirow{6}{*}{ 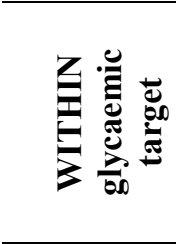 } & $\mathrm{D}$ & Upper limit of glycaemic target range & $\ldots$ & .. &. \\
\hline & $\mathrm{D}$ & Lower limit of glycaemic target range & $\ldots$ & $\ldots$ & $\ldots$ \\
\hline & $\mathrm{M}$ & Minimum recommended time to next measurement (TNM) & $\ldots$ & $\ldots$ & $\ldots$ \\
\hline & M & Maximum recommended TNM & $\ldots$ & $\ldots$ & $\ldots$ \\
\hline & $\mathrm{T}$ & Minimum recommended intravenous (IV) insulin infusion rate (IIR) ${ }^{[a]}$ & $\ldots$ & $\ldots$ & $\ldots$ \\
\hline & $\mathrm{T}$ & Maximum recommended IV IIR & $\ldots$ & $\ldots$ & $\ldots$ \\
\hline \multirow{6}{*}{ 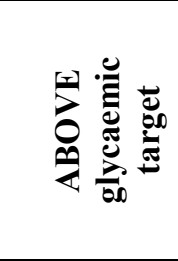 } & $\mathrm{O}$ & Is there a hyperglycaemic threshold instructing to inform medical staff? & $\ldots$ & $\ldots$ & $\ldots$ \\
\hline & $\mathrm{D}, \mathrm{O}$ & Hyperglycaemic threshold indicating when to inform medical staff & $\ldots$ & $\ldots$ & $\ldots$ \\
\hline & M & Minimum recommended TNM (h) & $\ldots$ & $\ldots$ & $\ldots$ \\
\hline & M & Maximum recommended TNM (h) & $\ldots$ & $\ldots$ & $\ldots$ \\
\hline & $\mathrm{T}$ & Minimum recommended IV IIR (U/h) & $\ldots$ & $\cdot$ &. \\
\hline & $\mathrm{T}$ & Maximum recommended IV IIR & $\ldots$ &. & $\ldots$ \\
\hline \multirow{9}{*}{ 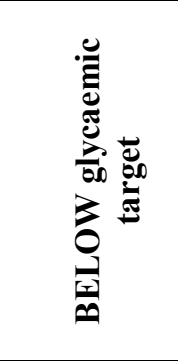 } & $\mathrm{O}$ & Is there a hypoglycaemic threshold instructing to inform medical staff? & . & & \\
\hline & $\mathrm{D}, \mathrm{O}$ & Hypoglycaemic threshold indicating when to inform medical staff & $\cdots$ & $\cdots$ & . \\
\hline & $\mathrm{D}, \mathrm{T}$ & Hypoglycaemic threshold indicating rescue glucose always & $\ldots$ & $\ldots$ & \\
\hline & M & Minimum recommended TNM if severe hypoglycaemia $(h)^{[b]}$ & $\ldots$ & $\ldots$ & $\ldots$ \\
\hline & M & Maximum recommended TNM if severe hypoglycaemia (h) & $\ldots$ & & \\
\hline & $\mathrm{T}$ & Minimum recommended IV IIR if severe hypoglycaemia $(\mathrm{U} / \mathrm{h})$ & $\ldots$ & $\ldots$ & . \\
\hline & $\mathrm{T}$ & Maximum recommended IV IIR if severe hypoglycaemia (U/h) & $\ldots$ & . & $\ldots$ \\
\hline & $\mathrm{T}$ & Minimum recommended IV glucose if severe hypoglycaemia (grams, g) & $\ldots$ & $\ldots$ & $\ldots$ \\
\hline & $\mathrm{T}$ & Maximum recommended IV glucose if severe hypoglycaemia (g) & $\ldots$ & $\ldots$ & $\ldots$ \\
\hline \multirow{10}{*}{ 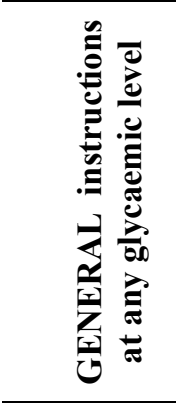 } & $\mathrm{D}, \mathrm{T}$ & Glycaemic threshold determining start of insulin infusion & $\ldots$ & $\cdots$ & $\ldots$ \\
\hline & M & Preferred monitoring method ${ }^{[\mathrm{c}]}$ & $\ldots$ & & $\ldots$ \\
\hline & M & Preferred monitoring blood sample ${ }^{[\mathrm{d}]}$ & $\ldots$ & $\ldots$ & $\ldots$ \\
\hline & $\mathrm{T}$ & Preferred insulin type ${ }^{[\mathrm{e}]}$ & $\ldots$ & $\ldots$ & $\ldots$ \\
\hline & $\mathrm{T}$ & Preferred insulin administration route ${ }^{[\mathrm{f}]}$ & $\ldots$ & $\ldots$ & $\ldots$ \\
\hline & $\mathrm{T}$ & Insulin titration method during admission ${ }^{[\mathrm{g}]}$ & $\ldots$ & $\ldots$ & $\ldots$ \\
\hline & $\mathrm{T}$ & Treatment actions depending on underlying condition or severity ${ }^{[\mathrm{h}]}$ & $\ldots$ & & \\
\hline & $\mathrm{T}$ & Treatment actions depending on concomitant medication ${ }^{[\mathrm{h}]}$ & $\ldots$ & & \\
\hline & $\mathrm{T}$ & Treatment actions if no feeding/glucose-based maintenance fluid & $\ldots$ & $\cdots$ & $\ldots$ \\
\hline & $\mathrm{O}$ & Is management after ICU discharge mentioned? & $\ldots$ & $\ldots$ & $\ldots$ \\
\hline \multirow{5}{*}{ 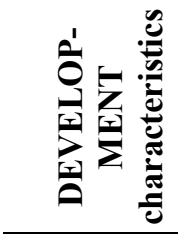 } & $\mathrm{P}$ & Are protocol development methods mentioned? & .. & $\ldots$ & . \\
\hline & $\mathrm{P}$ & Are protocol developers mentioned? & $\cdots$ & & \\
\hline & $\mathrm{P}$ & Is protocol evidence base mentioned? & $\ldots$ & $\ldots$ & $\ldots$ \\
\hline & $\mathrm{P}$ & Launch date / date of last update & $\ldots$ & $\ldots$ & $\cdots$ \\
\hline & & & $\cdots$ & $\cdots$ & $\cdots$ \\
\hline
\end{tabular}

* Instruction aim codes legend:
D: Diagnostic instructions
O: Organisational instructions
M: Monitoring instructions
P: Protocol development characteristics
T: Treatment instructions

[a] All insulin infusion rates of IV fast acting insulin in International Units per hour $(\mathrm{U} / \mathrm{h})$

[b] Severe hypoglycaemia defined as random blood glucose $\leq 2.2 \mathrm{mmol} / \mathrm{L}$ or symptomatic hypoglycaemia

[c] Blood Gas Analyser / Glucose Meter / Laboratory / Not Mentioned

[d] Arterial / Venous / Capillary / Not Mentioned

[e] Fast-acting / Fast-acting + Basal / Not mentioned

[f] Intravenous / Subcutaneous / Both intravenous and subcutaneous accepted / Not mentioned

[g] Fixed rates / Adjustments of previous rates

[h] Different IIR / Different TNM/ Mentioned as an aspect that should be considered / Not mentioned

[j] Data included as numbers, with one value per cell (i.e. per variable and protocol), representing either a numerical variable or the numerical code assigned to a categorical variable 
Table 2 - Non-quantifiable 'subjects' that could be present in protocols for glycaemic control, organised by instruction aim. Template table for future studies.

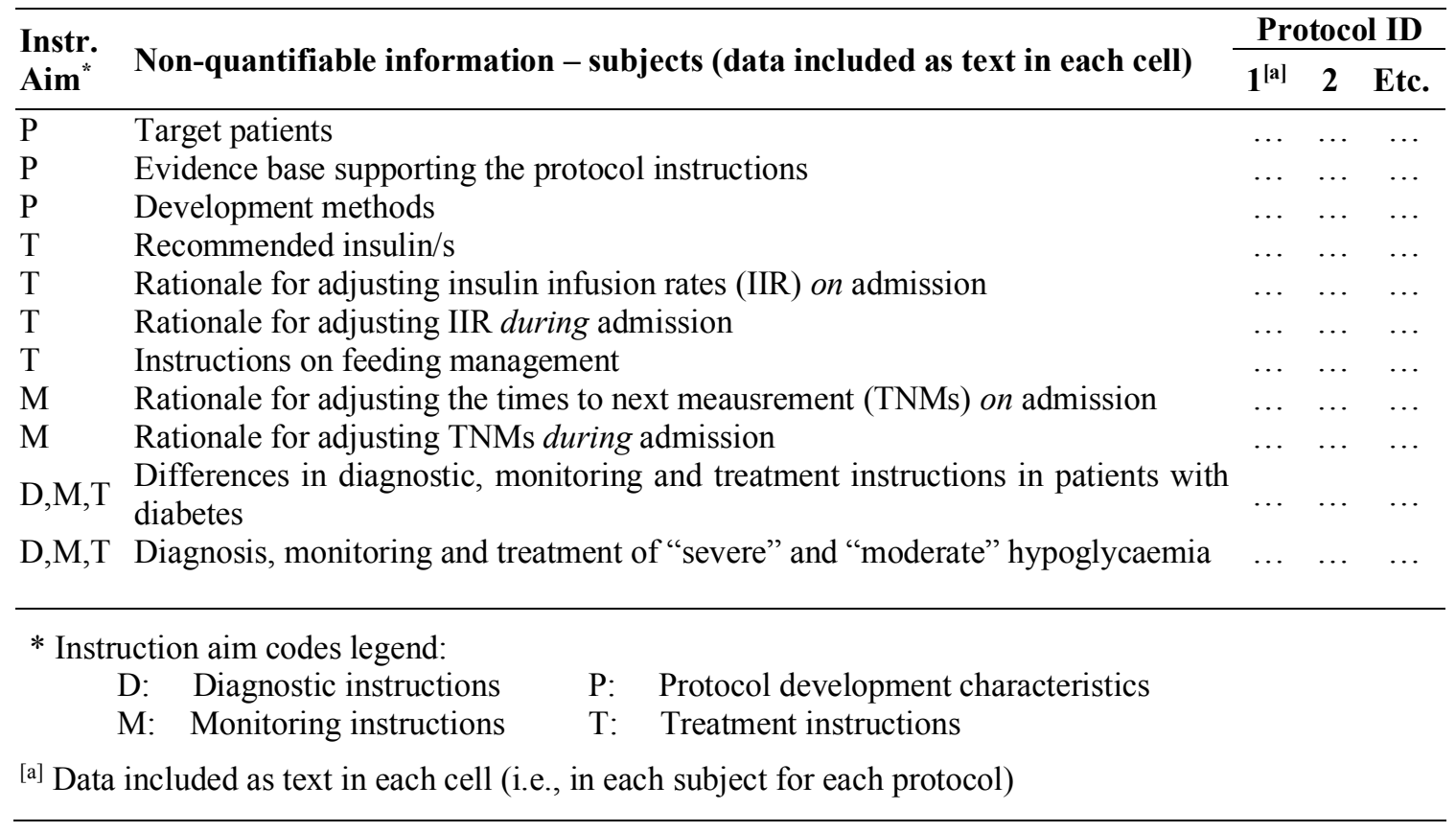


Table 3 - Numbers (total, median and range) and types of scenarios, processes and decisions included in the protocol/s for glycaemic control. Template table for future studies.

\begin{tabular}{lccc}
\hline & \multicolumn{2}{c}{ Protocol ID } \\
\cline { 2 - 4 } & $\mathbf{1}$ & $\mathbf{2}$ & Etc. \\
\hline Total number of scenarios per protocol & $\ldots$ & $\ldots$ & $\ldots$ \\
Median (range) number of processes per scenario & $\ldots$ & $\ldots$ & $\ldots$ \\
Median (range) number of decisions per scenario & $\ldots$ & $\ldots$ & $\ldots$ \\
Total number of glycaemic ranges considered per protocol & $\ldots$ & $\ldots$ & $\ldots$ \\
Are there any processes related to the following? (answer yes/no) & & & \\
Glycaemic monitoring frequency & $\ldots$ & $\ldots$ & $\ldots$ \\
Glycaemic monitoring methods (device/blood sample) & $\ldots$ & $\ldots$ & $\ldots$ \\
Insulin infusion rates & $\ldots$ & $\ldots$ & $\ldots$ \\
Insulin administration route & $\ldots$ & $\ldots$ & $\ldots$ \\
Rescue glucose infusion rates & $\ldots$ & $\ldots$ & $\ldots$ \\
Feeding assessment & $\ldots$ & $\ldots$ & $\ldots$ \\
Feeding rates & $\ldots$ & $\ldots$ & $\ldots$ \\
Calling medical staff / prescriber & $\ldots$ & $\ldots$ & $\ldots$ \\
Contacting diabetes team & $\ldots$ & $\ldots$ & $\ldots$ \\
Identifying cause of hypoglycaemia & $\ldots$ & $\ldots$ & $\ldots$ \\
Checking urine ketones & $\ldots$ & $\ldots$ & $\ldots$ \\
Considering patients' underlying condition or severity & $\ldots$ & $\ldots$ & $\ldots$ \\
Other [other fields can be added if needed] & $\ldots$ & $\ldots$ & $\ldots$ \\
Are there any decisions related to the following? (answer yes/no) & & & \\
Glycaemic monitoring frequency & $\ldots$ & $\ldots$ & $\ldots$ \\
Glycaemic monitoring methods (device/blood sample) & $\ldots$ & $\ldots$ & $\ldots$ \\
Insulin infusion rates & $\ldots$ & $\ldots$ & $\ldots$ \\
Insulin administration route & $\ldots$ & $\ldots$ & $\ldots$ \\
Rescue glucose infusion rates & $\ldots$ & $\ldots$ & $\ldots$ \\
Feeding assessment & $\ldots$ & $\ldots$ & $\ldots$ \\
Feeding rates & $\ldots$ & $\ldots$ & $\ldots$ \\
Calling medical staff / prescriber & $\ldots$ & $\ldots$ \\
Contacting diabetes team & $\ldots$ & $\ldots$ \\
Identifying cause of hypoglycaemia & $\ldots$ & $\ldots$ \\
Checking urine ketones & $\ldots$ & $\ldots$ \\
Considering patients' underlying condition or severity & $\ldots$ & $\ldots$ \\
Other [other fields can be added if needed] & $\ldots$ & $\ldots$ \\
\hline
\end{tabular}


Figure 1

Protocol name (date): XXX (XXX)

Blood glucose target range: $4-8.3 \mathrm{mmol} / \mathrm{L}$

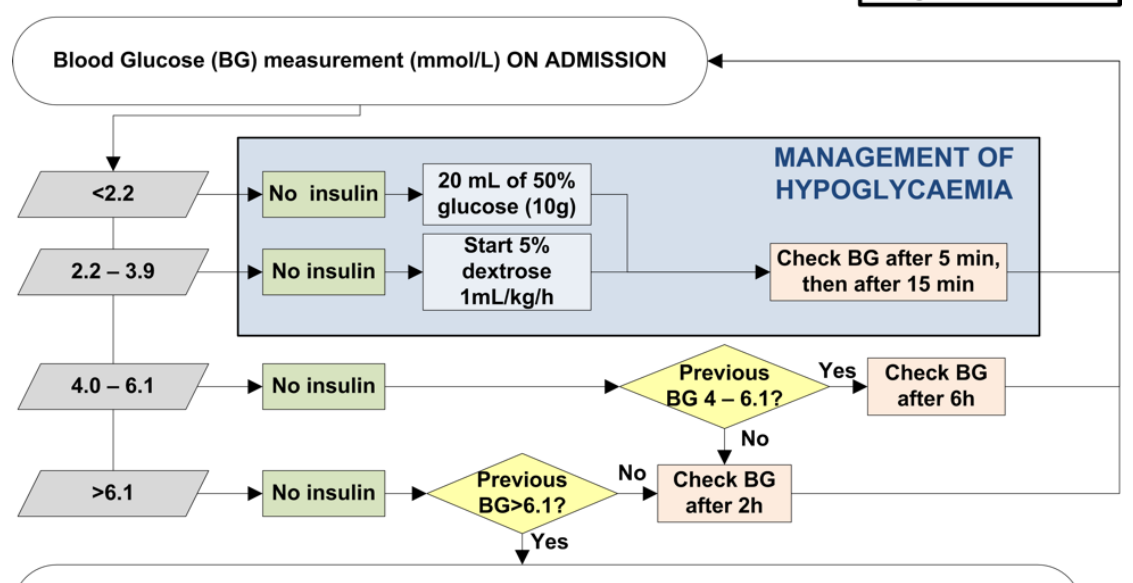

Blood Glucose (BG) measurement (mmol/L) AFTER commencement of treatment for hyperglycaemia

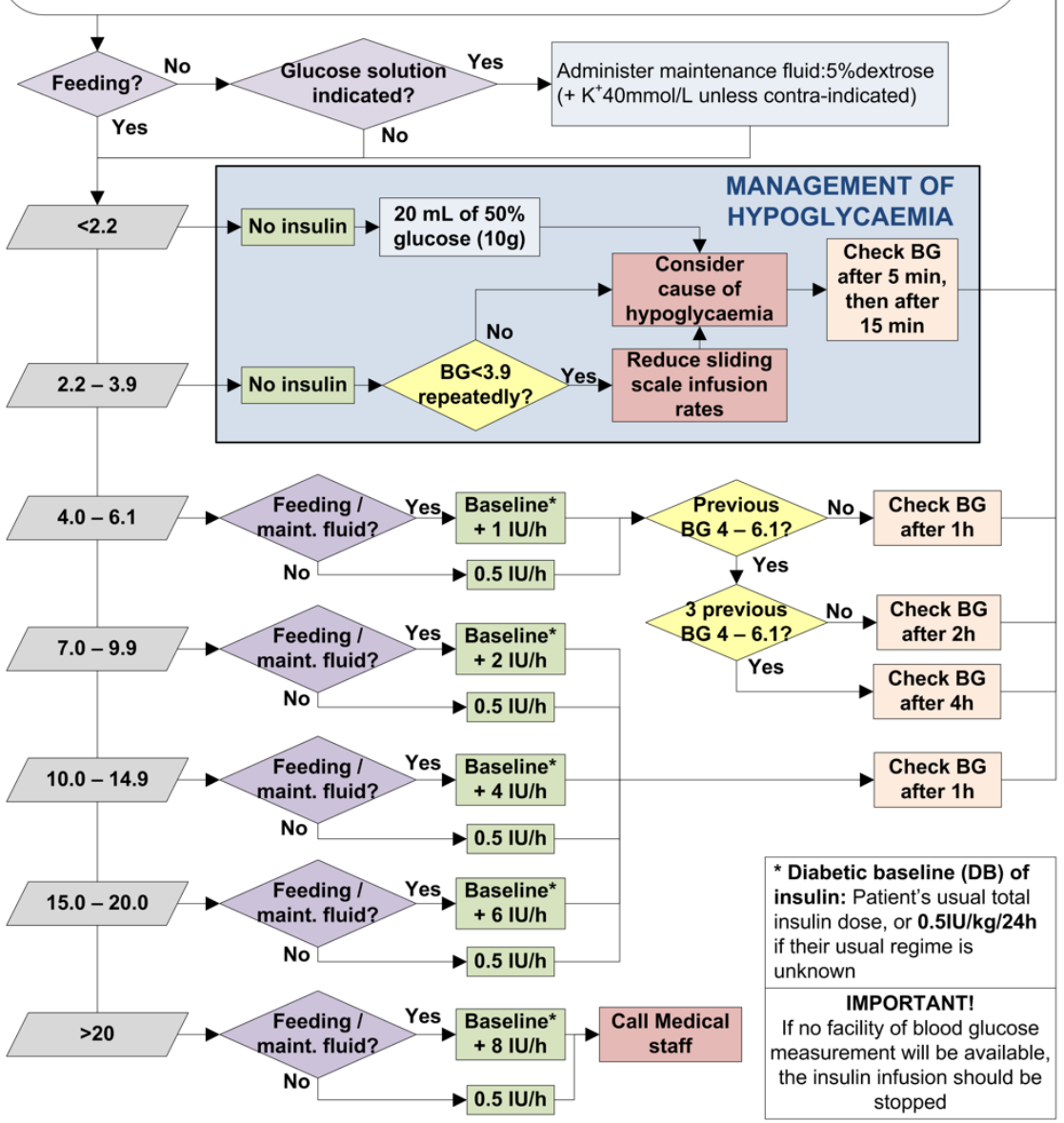

Glycaemic measurements delimiting the start/end of one scenario (loop)

Ranges of glycaemic levels

Treatment processes on insulin infusion rates (international units per hour)

Monitoring processes on times to next measurement (hours)

Treatment processes on management of hypoglycaemia

Organisational processes on consulting with other/medical staff

Diagnostic decisions on stability/instability of glycaemic levels

Treatment decisions on feeding or maintenance fluids 
Figure 2
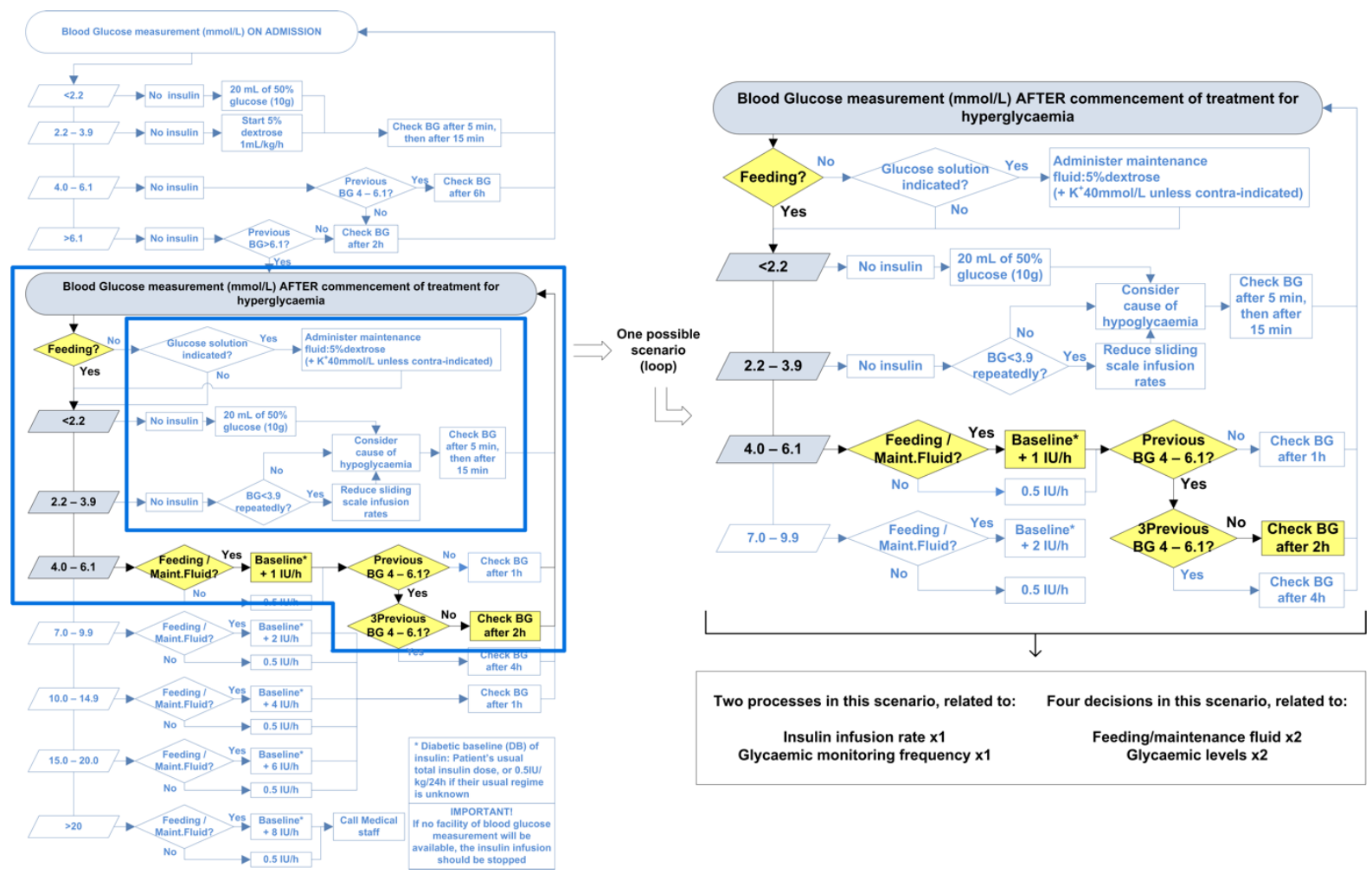